\title{
SWITCHABLE ALUMINUM NITRIDE MEMS RESONATOR USING PHASE CHANGE MATERIALS
}

\author{
G. Hummel, Y. Hui, Z. Qian, and M. Rinaldi \\ Northeastern University, Boston, Massachusetts, USA
}

\begin{abstract}
This paper reports on the first demonstration of a reconfigurable Aluminum Nitride (AIN) piezoelectric Micro Electro Mechanical Systems (MEMS) resonator using phase change material (PCM) based switchable electrodes, enabling switching and reconfiguration of a high quality factor $(Q \sim 1400)$ and high frequency $(\sim 260 \mathrm{MHz})$ piezoelectric micro-acoustic resonator. This innovative design solution allows direct control and reconfigurability of the electrical coupling across the piezoelectric body of the device which enables effective ON/OFF switching of the acoustic resonance ( $18 X$ impedance variation at resonance) and reconfiguration of the device electromechanical coupling $(0 \%$ $\left.<k_{t}^{2}<0.7 \%\right)$ and electrical capacitance $\left(258 f F<C_{0}<340 f F\right)$ which can potentially lead to the implementation of filter architectures whose frequency, order, bandwidth, and roll-off can be dynamically reconfigured.
\end{abstract}

\section{INTRODUCTION}

In recent years the demand for highly reconfigurable radio frequency (RF) systems, capable of operating in the severely crowded and rapidly changing modern commercial and military spectral environment, at a reduced overall component count and with a reduced development cost compared to conventional multiband radios, has been steadily growing. In this context, the implementation of high quality factor, $Q$, micro acoustic resonators with monolithically integrated switching and frequency reconfiguration functionalities will dramatically reduce loss associated with the filtering element enabling new radio architectures with enhanced spectrum coverage, whose implementation is currently prevented by the lack of such high performance and intrinsically reconfigurable components.

High Q MEMS resonant devices enable the implementation of low insertion loss filters in a very small form factor. Different MEMS resonator technologies based on electrostatic [1-2] or piezoelectric [3-4] transduction have been investigated. Among these, the piezoelectric Aluminum Nitride (AIN) contour-mode resonator (CMR) technology [3, 5, 6] has emerged as one of the most promising solutions in enabling the fabrication of multiple frequency and high performance resonators on the same silicon chip. Nevertheless, the current filtering solutions based on AIN micro acoustic resonant devices cannot be dynamically reconfigured to operate at different frequencies, orders, and bandwidths.

Switching of piezoelectric resonance in AlN contour-mode resonators has been recently demonstrated by integrating a MEMS capacitive switch over the AlN piezoelectric film [7]. This work can be considered the first demonstration of the monolithic integration of phase change material (PCM) RF switches with a MEMS resonator technology to implement switching and reconfiguration functionalities. In particular, switching and reconfiguration of a high quality factor $(Q \sim 1400)$ and high frequency $(\sim 260 \mathrm{MHz})$ piezoelectric micro-acoustic resonator is demonstrated thanks to the unprecedented monolithic integration of PCM switches with the AlN piezoelectric MEMS resonator technology.

Phase change materials are chalcogenide materials that show a significant change in resistivity between the amorphous (OFF) and crystalline (ON) states. Reversible switching behavior can be achieved by applying low voltage pulses of proper duration (direct heating) across the PCM [9]. Due to this property, PCMs have been investigated for use as Radio Frequency (RF) switches [9]. IN this work, miniaturized $\left(2 \times 2 \mu m^{2}\right) G_{50} \mathrm{Te}_{50} \mathrm{PCM}$ vias are employed as low loss (ON resistance $<5 \Omega$ ), high dynamic range (ON/OFF ratio $\sim 10^{7}$ ), and low OFF-capacitance ( $\left.\sim 40 f F\right)$ ohmic switches to control and reconfigure the electrical connections of the interdigital electrode employed to excite vibration in a piezoelectric resonator (Fig. 1, 2), enabling effective ON/OFF switching of the acoustic resonance $(\sim 18 \mathrm{X}$ impedance variation at resonance) and reconfiguration of the device electromechanical coupling $\left(0 \%<k_{t}^{2}<0.7 \%\right)$ and electrical capacitance.

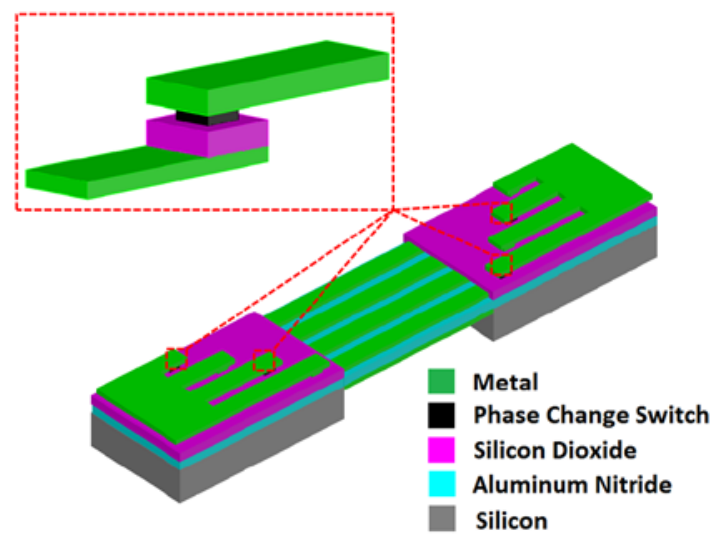

Figure 1: 3D representation of the switchable and reconfigurable resonator with closer view of an individual PCM via switch.

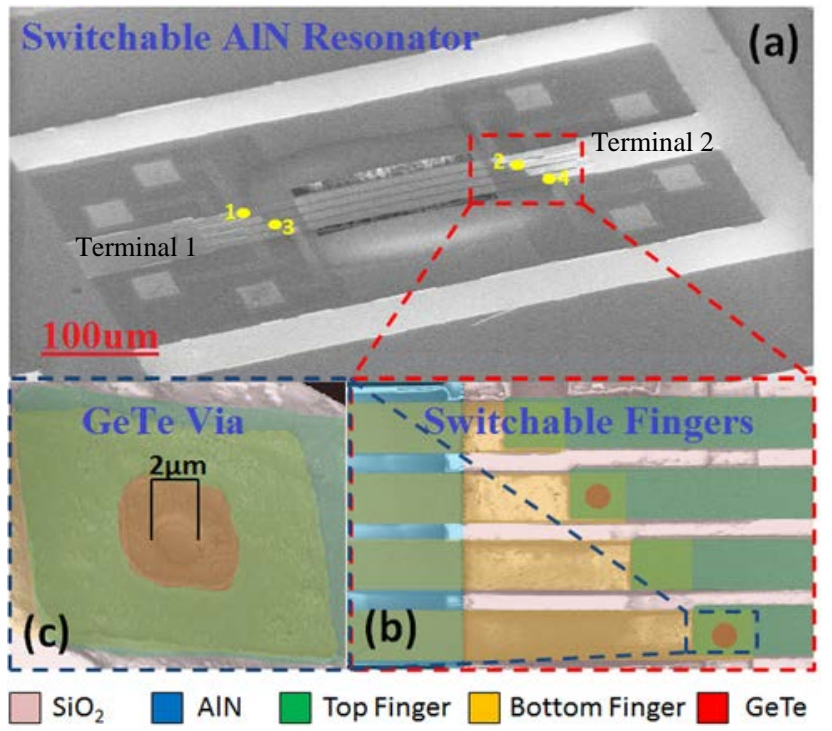

Figure 2: SEM Images of (a) the switchable and reconfigurable resonator (the locations of the PCM via switches utilized in this work are highlighted in gold), (b) close-up of phase change switches integrated into the fingers of the resonator, and (c) individual PCM via switch. 
This innovative technology has the potential to deliver a new class of monolithically integrated and highly reconfigurable RF components such as resonators, filters, and capacitors capable of achieving the highest level of programmability with the minimum possible effect on the performance (by minimizing the number of physically separated RF components), enabling new radio architectures with enhanced spectrum coverage. This new family of RF components can be fabricated with a relatively simple 6mask fabrication process (instead of the 10-mask required for the capacitive switchable electrodes) and does not require the creation of a micro/nano scale gap between the resonator and the electrodes for the switching mechanism, which significantly reduces the complexity of the fabrication process.

Furthermore, thanks to the unique reversible switching behavior of PCMs, low voltages (1-2.5V compared to $35-40 \mathrm{~V}$ in the electrostatic case) are required for switching and, differently from conventional MEMS capacitive switch technologies that have been integrated with AIN resonators previously [7], PCM switches do not need to be powered to maintain either state.

\section{DESIGN AND FABRICATION}

A conventional static contour-mode resonator is composed of an AlN film sandwiched between two metal electrodes (Fig. 1). When an alternating current (ac) signal is applied across the thickness $T$ of the AlN film, a contour-extensional mode of vibration is excited through the equivalent $d_{31}$ piezoelectric coefficient of AlN. Given the equivalent mass density, $\rho_{\text {eq }}$, and Young's modulus, $E_{e q}$, of the material stack that forms the resonator, the center frequency, $f_{0}$, of this laterally vibrating mechanical structure is set by the period, $W$, of the interdigital electrode patterned on top of the AlN plate and can be approximately expressed as:

$$
f_{0}=\frac{1}{2 W} \sqrt{\frac{E_{e q}}{\rho_{e q}}}
$$

For a given geometry of the AIN resonant micro-plate and period of the interdigital electrode, the equivalent electrical impedance of the device is set by the number of metal fingers, $n$, composing the interdigital electrode [5-7]. In particular, only the fraction of the device area covered by the metal fingers is effectively employed for transduction. Therefore, the device electrical static capacitance, $C_{0}$, and electromechanical coupling coefficient, $k_{t}{ }^{2}$, are directly proportional to the number of metal fingers, $n$, composing the interdigital electrode employed to excite the higher order $\left(n^{\text {th }}\right)$ contour-extensional mode of vibration in the AlN micro-plate.

In this work, an innovative design solution, that enables dynamic reconfiguration of the number of metal fingers composing the interdigital electrode employed to excite a higher order contour-extensional mode of vibration in an AlN resonant microplate, is introduced for the first time. This new approach allows direct control and reconfigurability of the electrical coupling across the piezoelectric body of the device, enabling effective ON/OFF switching of the acoustic resonance (OFF state corresponding to $n=0$ ) and reconfiguration of the device electromechanical coupling coefficient and electrical static capacitance.

The resonant core of this innovative device concept is composed of a $500 \mathrm{~nm}$ thick AlN layer sandwiched between a bottom electrically floating plate electrode and a top interdigital electrode composed of $n=4$ metal fingers. Each metal finger completely covers the resonant body of the device extending up to the anchoring regions where it is overlapped by the electrical terminal of the resonator, but separated by a $\mathrm{SiO}_{2}$ insulating layer. 4 miniaturized $\left(2 \times 2 \mu \mathrm{m}^{2}\right)$ Phase Change Material (PCM) vias are monolithically integrated with the resonant structure and employed as low loss radio frequency (RF) switches to connect each of the 4 metal fingers forming the device interdigital electrode to the electrical terminals of the resonator through the $\mathrm{SiO}_{2}$ insulating layer (Fig. 1, 2). $\mathrm{Ge}_{50} \mathrm{Te}_{50}$ is chosen to implement the PCM via switches due to its high ON/OFF ratio $\left(\sim 10^{6}\right)$ and low loss at radio frequencies [9]. The transition temperature (ON/OFF switching) of each PCM via is readily reached by passing current through the PC material itself (direct heating).

When all the vias are in the OFF state, the terminals of the device are ideally completely isolated (open circuit) and no electric field is coupled across the piezoelectric material (hence no resonance is excited). In practice, a high impedance path between the two terminals is formed through substrate parasitics $\left(C_{p}, R_{p}\right.$, $R_{p p}$ ) and the capacitance and resistance associated with the combination of PCM via switches in the OFF state $\left(C_{\text {switch }}, R_{\text {switch }}\right)$ (Fig. 3-a).

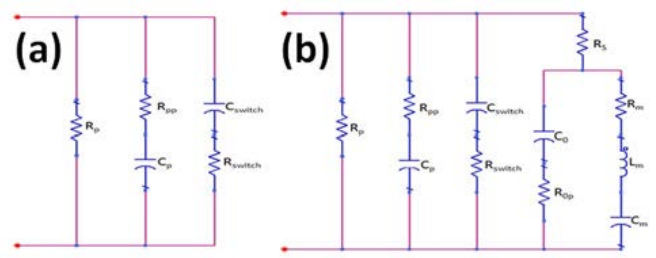

Figure 3: Equivalent circuit model of the device in the (a) OFF state, and (b) State 1 and State 2. $C_{p}, R_{p}$, and $R_{p p}$ represent the parasitics. $C_{\text {switch }}$ and $R_{\text {switch }}$ are the capacitance/resistance associated with the combination of PCM via switches in the OFF state. $R_{S}$ is the loss introduced by the combination of PCM vias in the ON state - note that $R_{S}$ has minimal effect on resonator performance. $C_{0}$ and $R_{0 p}$ are static capacitance/resistance of the piezoelectric transducer. $R_{m}, C_{m}$, and $L_{m}$ represent the motional branch of the resonator. All values used for these equivalent circuit elements are reported in Table 1.

When two vias (i.e. vias 1 and 4 in Figure 2) are in the $O N$ state (State 1), only two fingers are connected to form the interdigital electrode with a polarity that uniquely matches the one of the strain field for the $4^{\text {th }}$ order contour-extensional mode of vibration of the plate. Therefore, a $4^{\text {th }}$ order contour-extensional mode of vibration is excited in the AlN micro-plate by means of a lateral field excitation scheme [5-6, 8]. Such configuration results in a relatively high impedance resonance due to the low values of device static capacitance, $C_{0}$, and electromechanical coupling coefficient, $k_{t}^{2}$, associated with the 2-finger top interdigital electrode configuration for which only a fraction of the device area (the one covered by the two metal fingers) is effectively employed for transduction.

Table 1: Values of the equivalent circuit elements for all the states as pictured in Figure 6. Note that the series resistance, $R_{S}$, introduced by the PCM vias has a minimal effect on the resonator performance. Values of parasitic components were extracted from the data using layout considerations. In the State 2 model, the malfunctioning PCM via 2 was considered to be OFF.

\begin{tabular}{|c|c|c|c|c|c|c|c|c|c|c|c|}
\hline \multirow[t]{2}{*}{ States } & \multicolumn{11}{|c|}{ Equivalent Circuit Element Values } \\
\hline & $R_{m}$ & $C_{m}$ & $L_{m}$ & $R_{S}$ & $R_{0 p}$ & $C_{0}$ & $R_{\text {switch }}$ & $C_{\text {switch }}$ & $C_{p}$ & $R_{p p}$ & $R_{p}$ \\
\hline OFF & -- & -- & -- & -- & -- & -- & $0 \Omega$ & $69.232 \mathrm{fF}$ & $75.768 \mathrm{fF}$ & $47.095 \mathrm{k} \Omega$ & $48.04 \Omega$ \\
\hline State 1 & $422.8 \Omega$ & $1.0102 \mathrm{fF}$ & $376.48 \mu \mathrm{H}$ & $5 \Omega$ & $5 \Omega$ & $258.17 \mathrm{fF}$ & $5 \Omega$ & $86.092 \mathrm{fF}$ & $75.768 \mathrm{fF}$ & $47.095 \mathrm{k} \Omega$ & $48.04 \Omega$ \\
\hline State 2 & $227.6 \Omega$ & $1.9437 \mathrm{fF}$ & $195.88 \mu \mathrm{H}$ & $3.75 \Omega$ & $5 \Omega$ & $359.99 \mathrm{fF}$ & $3.75 \Omega$ & $106.79 \mathrm{fF}$ & $75.768 \mathrm{fF}$ & $47.095 \mathrm{k} \Omega$ & $48.04 \Omega$ \\
\hline
\end{tabular}


When three vias (i.e. vias 1,3 , and 4 in Figure 2) are in the $O N$ state (corresponding to a 3-finger interdigital electrode configuration), the effective transduction area for $4^{\text {th }}$ order contourextensional mode of vibration is increased resulting in larger values of device static capacitance, $C_{0}$, and electromechanical coupling coefficient, $k_{t}^{2}$, hence, lower impedance resonance. Maximum transduction area, $C_{0}$, and $k_{t}^{2}$, hence minimum impedance resonance, are achieved when all 4 vias are in the $O N$ state, forming a 4-finger interdigital electrode with a polarity that uniquely matches the one of the strain field for the $4^{\text {th }}$ order contour-extensional mode of vibration of the plate. The values of $C_{0}$ and $k_{t}^{2}$, estimated by Finite Element Method (FEM) simulation using COMSOL Multiphysics, for the four possible device configurations are reported in Figure 4.

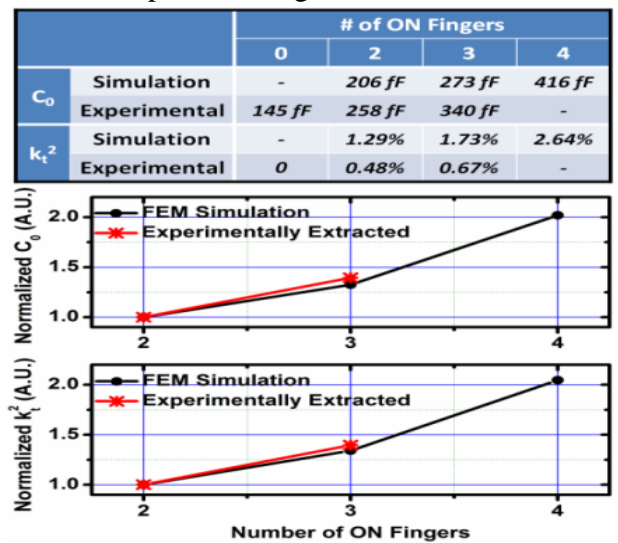

Figure 4: Comparison between Finite Element Method (FEM) simulated (COMSOL) and experimentally extracted values of $C_{0}$ and $k_{t}^{2}$ for the different possible device states.

The switchable resonator presented in this work was fabricated using a relatively simple 6-mask post-CMOS compatible fabrication process shown in Figure 5.
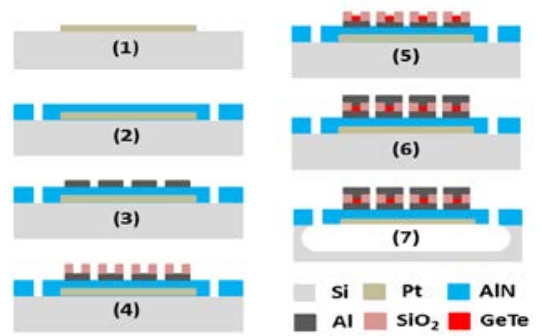

Figure 5: Device fabrication process. (1) Platinum electrode, (2) Aluminum nitride deposition and etch, (3) Aluminum fingers, (4) PECVD oxide deposition and etch, (5) Phase change material deposition, (6) Top Aluminum contact, (7) $\mathrm{XeF}_{2}$ release.

The fabrication process began with a high resistivity $\mathrm{Si}$ substrate (resistivity $>10,000 \Omega \cdot \mathrm{cm}$ ). A $5 \mathrm{~nm} / 95 \mathrm{~nm}$ Titanium/Platinum (Ti/Pt) layer was sputter deposited and patterned with a lift-off process to form the bottom electrically floating electrode. Next, a high quality c-axis oriented 500 nm Aluminum Nitride (AIN) layer was sputter deposited on top of the $\mathrm{Ti} / \mathrm{Pt}$ layer. Inductively Coupled Plasma (ICP) etching in $\mathrm{Cl}_{2}$ based chemistry was used to open vias to the bottom Pt and define the dimensions of the micro-plate resonator. Next, sputter deposition was used to deposit a $100 \mathrm{~nm}$ layer of Aluminum (Al) which was patterned using lift-off to create the interdigital electrodes on top of the AlN micro-plate. Plasma Enhanced Chemical Vapor Deposition (PECVD) was used to deposit 300nm of $\mathrm{SiO}_{2}$ to form the insulation layer for the PCM switches. $2 \times 2 u^{2}$ vias were etched in the $\mathrm{SiO}_{2}$ using ICP with $\mathrm{CHF}_{3}$ based chemistry. DC Pulse Sputtering was used to deposit $100 \mathrm{~nm} / 10 \mathrm{~nm}$ of $\mathrm{Ge}_{50} \mathrm{Te}_{50} / \mathrm{Ti}$ in the vias and pattern using a lift-off process. A $100 \mathrm{~nm}$ Al film was deposited using sputter and patterned with lift-off to form the top probing pad and the top electrode of the PCM switches. Finally, Xenon Difluoride $\left(\mathrm{XeF}_{2}\right)$ isotropic etching was used to etch the Si substrate and create an air gap under the resonator, completely releasing the resonant structure.

\section{EXPERIMENTAL RESULTS AND ANALYSIS}

The electrical response of the fabricated switchable MEMS resonator was measured by an Agilent E5071C network analyzer after performing an open-short-load calibration on a standard substrate. The transition temperature, needed for ON/OFF switching of the PCM vias, was reached by passing current through the PC material itself (direct heating). ON state was achieved by applying a $300 \mu$ s pulse with amplitude of $1 \mathrm{~V}$ and a rise/fall time of 100 ns while the OFF state was achieved by applying a $4 \mu \mathrm{s}$ pulse with amplitude of $2.5 \mathrm{~V}$ and a rise/fall time of $5 \mathrm{~ns}$. The device was reconfigured to operate in 3 different states: OFF (all vias in the OFF state), State 1 (vias 1 and 4 in the $O N$ state), and State 2 (all vias 1-4 in the $O N$ state) (Fig. 6). Although all the PCM vias were turned $O N$ in State 2, via 2 malfunctioned, showing a resistance of $\sim 140 M \Omega$. Therefore, only 3 fingers (vias 1 , 3 , and 4) were effectively connected to form the interdigital electrode.

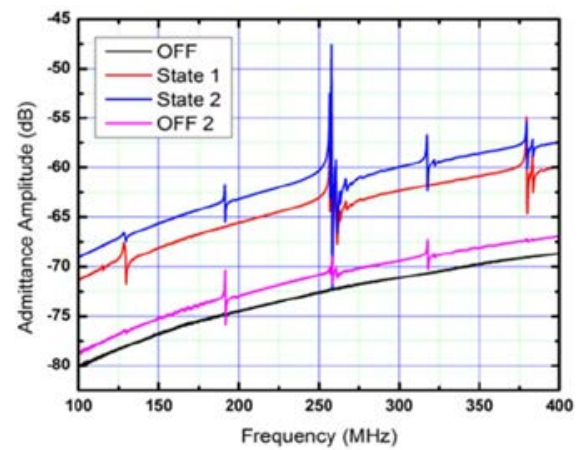

Figure 6: Measured admittance of the resonator in the OFF state (all the PCM vias OFF); State 1 (vias 1 and 4 ON); State 2 (all the vias ON); OFF 2 (all the vias OFF again). The difference between OFF and OFF 2 is believed to be due to imperfect reamorphization of the PCM vias. In State 2, via 2 functioned imperfectly, connecting the metal finger to the terminal with a resistance of $\sim 140 M \Omega$. Therefore, only 3 fingers were effectively connected to form the interdigital electrode.

The measured responses of the device in the 3 different states were fitted to the equivalent circuits in Figure 3. The substrate parasitics $\left(C_{p}, R_{p}\right.$, and $\left.R_{p p}\right)$ and the capacitance $\left(\mathrm{C}_{\text {switch }}\right)$ and resistance $\left(R_{\text {switch }}\right)$, associated with the combination of PCM vias in the $O F F$ state were extracted from the OFF state measurement (Fig. 3-a) while the remaining equivalent circuit components were extracted from State 1 and State 2 measurements (Fig. 3-b) (Being: $R_{S}$ - the loss introduced by the combination of PCM vias in the $O N$ state; $C_{0}$ and $R_{0 p}$ - static capacitance and resistance of the piezoelectric transducer; and $R_{m}, C_{m}$, and $L_{m}$ - the motional branch of the resonator). The results of the fitting procedure are reported in Figures 7-8 and Table 1.

Values of $O F F$ resistance larger than $\sim 250 M \Omega$, ON resistance of $\sim 5 \Omega\left(O N / O F F\right.$ ratio of $\left.\sim 10^{7}\right)$ and OFF capacitance of $\sim 40 f F$, were extracted for the PCM vias. 


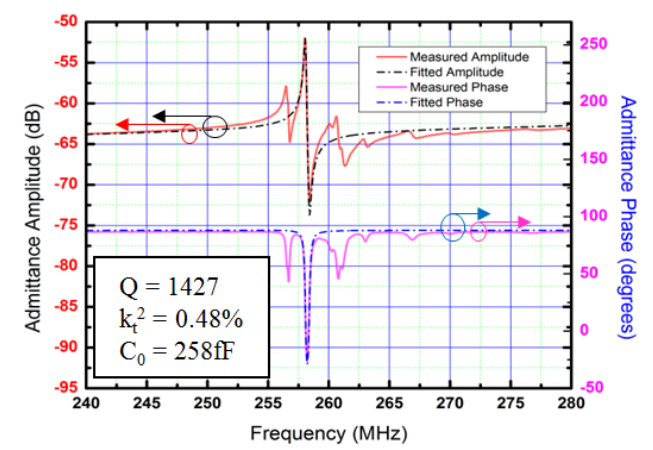

Figure 7: Measured admittance and circuit model fitting (Fig. 3-b, Table 1) of the fabricated resonator with PCM vias 1 and 4 in the ON state (State 1).

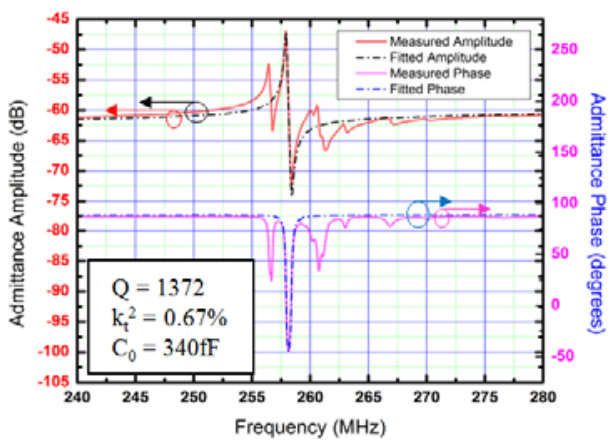

Figure 8: Measured admittance and circuit model fitting (Fig. 3-b, Table 1) of the fabricated resonator with the all 4 PCM vias in the ON state (State 2). Although all the PCM vias are turned ON, via 2 malfunctioned, showing a resistance of $\sim 140 M \Omega$. Therefore, only 3 fingers are effectively connected to form the interdigital electrode.

The values of $C_{0}$ and $k_{t}^{2}$ extracted from the measurements for each possible device configuration were compared with the ones estimated by FEM simulations (Fig. 4). Although a slight difference in absolute values is observed due to imperfections in the model and material coefficients used in the simulation, the experimentally recorded relative variations of $C_{0}$ and $k_{t}^{2}$, for different configurations of the top electrode, closely match the ones achieved by FEM simulations.

These experimental results clearly indicate that the proposed design solution enables not only effective ON/OFF switching of the acoustic resonance of the device ( $\sim 18 X$ impedance variation at resonance, $C_{\text {on }} / C_{\text {off }} \sim 4$ with parasitics, $C_{\text {on }} / C_{\text {off }} \sim 7$ without parasitics), but also tunability of the electrical capacitance (258fF $\left.<C_{0}<340 f F\right)$ and reconfiguration of the device electromechanical coupling $\left(0 \%<k_{t}^{2}<0.7 \%\right)$ (Figures $4,6-8$, Table 1$)$ which can potentially lead to the implementation of filter architectures (exclusively based on AIN/PCM high performance resonators and capacitors) whose frequency, order, bandwidth, and roll-off can be dynamically reconfigured.

\section{CONCLUSION}

This paper reports on first demonstration of the monolithic integration of phase change material (PCM) RF switches with a MEMS resonator technology to implement switching and reconfiguration functionalities. A reconfigurable piezoelectric MEMS resonator using phase change material (PCM) based switchable electrodes was demonstrated. Miniaturized $\left(2 \times 2 \mu \mathrm{m}^{2}\right)$ $\mathrm{Ge}_{50} \mathrm{Te}_{50}$ vias were employed as low loss (ON resistance $<5 \Omega$ ), high dynamic range (ON/OFF ratio $\sim 10^{7}$ ), and low OFFcapacitance $(\sim 40 f F)$ ohmic switches to control and dynamically reconfigure the number of metal fingers composing the interdigital electrode employed to excite a higher order contour-extensional mode of vibration in an AlN micro-plate. This innovative design solution allowed direct control and reconfigurability of the electrical coupling across the piezoelectric body of the device (by selecting the number of metal lines forming the electrode) enabling not only effective $O N / O F F$ switching of the acoustic resonance ( $\sim 18 X$ impedance variation at resonance), but also reconfiguration of the device electromechanical coupling $\left(0 \%<k_{t}^{2}<0.7 \%\right)$ and electrical capacitance $\left(258 \mathrm{fF}<C_{0}<340 \mathrm{fF}\right.$ ) which can potentially lead to the implementation of filter architectures (exclusively based on AlN/PCM high performance resonators and capacitors) whose frequency, order, bandwidth, and roll-off can be dynamically reconfigured.

\section{ACKNOWLEDGEMENTS}

The authors would like to thank the staff of the George J. Kostas Nanotechnology and Manufacturing Facility, Northeastern University (Scott McNamara and David McKee) for their support in device fabrication. The authors would also like to acknowledge DARPA MTO for funding (Dr. William Chappell).

\section{REFERENCES}

[1] C. T.-C. Nguyen, "MEMS technology for timing and frequency control,” IEEE Trans. Ultrason. Ferroelectr. Freq. Control, vol. 54, no. 2, pp. 251-270, Feb. 2007.

[2] D. Weinstein and S. A. Bhave, "Internal dielectric transduction of a $4.5 \mathrm{GHz}$ silicon bar resonator," in IEEE Int. Electron Devices Meeting, Dec. 2007, pp. 415-418.

[3] G. Piazza, P. J. Stephanou, and A. P. Pisano, "Piezoelectric aluminum nitride vibrating contour-mode MEMS resonators," J. Microelectromech. Syst., vol. 15, no. 6, pp. 1406-1418, Dec. 2006.

[4] R. Abdolvand, G. K. Ho, J. Butler, and F. Ayazi, "ZnO-onnanocrystalline-diamond lateral bulk acoustic resonators,” in Proc. $20^{\text {th }}$ IEEE Int. Conf. Micro Electro Mechanical Systems, Kobe, Japan, Jan. 2007, pp. 795-798.

[5] M. Rinaldi, C. Zuniga, C. Zuo, and G. Piazza, "Super High Frequency Two-Port AlN Contour-Mode Resonators for RF Applications”, IEEE Transactions on Ultrasonics, Ferroelectrics, and Frequency Control, vol. 57, n. 1, pg. 3845, 2010.

[6] M. Rinaldi, C. Zuo, J. Van der Spiegel and G. Piazza, "Reconfigurable CMOS Oscillator based on Multi-Frequency AlN Contour-Mode MEMS Resonators”, IEEE Transactions on Electron Devices, vol. 58, issue 5, pg. 1281-1286, 2011.

[7] Nordquist, C.D.; Olsson, R.H.; Scott, S.M.; Branch, D.W.; Pluym, T.; Yarberry, V., "On/Off micro-electromechanical switching of AlN piezoelectric resonators," Microwave Symposium Digest (IMS), 2013 IEEE MTT-S International , vol., no., pp.1,4, 2-7 June 2013

[8] Piazza, Gianluca. "Chapter 2: Contour Mode Aluminum Nitride Piezoelectric MEMS Resonators and Filters." MEMSbased Circuits and Systems for Wireless Communication. Ed. Christian Enz and Andreas Kaiser. New York: Springer, 2013. 29-37. Print.

[9] Y. Shim, G. Hummel, and M. Rais-Zadeh, "RF switches using phase change materials," IEEE International Conference on Microelectromechanical Systems (MEMS'13), Taipei, Taiwan, pp. 237-240, Jan, 2013.

\section{CONTACT}

*G. Hummel, tel: +1-603-762-3014; hummel.gw@husky.neu.edu 